\title{
How can we address the controversies surrounding the use of NSAIDS in neurodegeneration?
}

\author{
"We propose developing transgenic mouse and hybrid enzyme \\ constructs as a research platform to help further our understanding \\ of NSAIDs and their potential application in this pathology."
}

First draft submitted: 25 March 2016; Accepted for publication: 11 May 2016; Published online: 30 June 2016

Keywords: COX-1-10aa-PGIS • COX-2-10aa-mPGES-1 • cyclooxygenase • hybrid enzyme - neurodegeneration $\bullet$ neuroinflammation $\bullet$ nonsteroidal anti-inflammatory drugs $\bullet$ NSAIDs - PGE2 • PGI2

Neuroinflammation, which is regulated by arachidonic acid (AA) metabolites, is a key factor in the development of neurodegenerative disorders (NDD) [1-3]. Accordingly, anti-inflammatory agents, such as NSAIDs have been suggested as pharmacotherapy to prevent and treat several NDD, such as Alzheimer's disease, Parkinson's disease and amyotrophic lateral sclerosis [2-5]. However, the beneficial effects of these treatments have only exhibited in the preclinical phase, and they do not elicit similar effects in all patients with NDD [4]. Some clinical trial results even displayed detrimental effects on neuronal function, with few preventive benefits on neuronal degeneration [5-7].

The diverse end-active prostanoids including prostaglandin $(\mathrm{PG}) \mathrm{E}_{2}\left(\mathrm{PGE}_{2}\right)$, prostacyclin $\left(\mathrm{PGI}_{2}\right), \mathrm{PGD}_{2}, \mathrm{PGF}_{2}$ and thromboxane $\mathrm{A}_{2}$ $\left(\mathrm{TXA}_{2}\right)$ are produced from the metabolism of AA by the coupling reactions of the upstream COX-1/-2 and downstream individual prostanoid synthases. In particular, the $\mathrm{PGE}_{2}$ synthesized by inducible COX-2 coupled to microsomal $\mathrm{PGE}_{2}$ synthase-1 (mPGES-1) activates its receptors to display neurotoxic activity [8-11]. However, the $\mathrm{PGI}_{2}$ synthesized by both COX-1 and COX-2 coupled to prostacyclin synthase has neuroprotective activ- ity [12-14]. NSAIDs inhibiting the upstream COX-1 and COX-2 to nonspecifically reduce all of the prostanoid biosyntheses are the most widely used drugs worldwide. For example, approximately 40,000 tons of aspirin, a type of NSAID, are produced globally per year as a therapeutic or preventive treatment for a variety of illnesses, including cardiovascular disease [15] and pain relief. Thus, it is important to understand the molecular and cellular mechanisms underlying toxic and beneficial effects of the NSAIDs on NDD, and also to develop pharmacotherapies which could improve the current NSAIDs through targeting more specific downstream enzymes to aid in the prevention and treatment of NDD.

Recent work by our lab showed that transgenic mice expressing an engineered hybrid enzyme showed altered AA metabolism, skewed toward $\mathrm{PGI}_{2}$ production. This, in turn, reduced the conversion of $\mathrm{AA}$ metabolites to inflammatory PGE $_{2}$ and TXA 2 [14,16-19]. As adults, these animals exhibited improved cognitive performance in behavioral studies [14]. This model has also been shown to be effective in other diseases linked to ischemia and inflammation [14,18]. Additionally, preliminary data suggested that these mice have a longer
Qing-lan Ling

The Center for Experimental Therapeutics \& Pharmacoinformatics, Department of Pharmacological \& Pharmaceutical Sciences, College of Pharmacy, University of Houston, Houston, TX 77204, USA

\section{Emma Murdoch}

The Center for Experimental Therapeutics \& Pharmacoinformatics, Department of Pharmacological \& Pharmaceutical Sciences, College of Pharmacy, University of Houston, Houston, TX 77204, USA

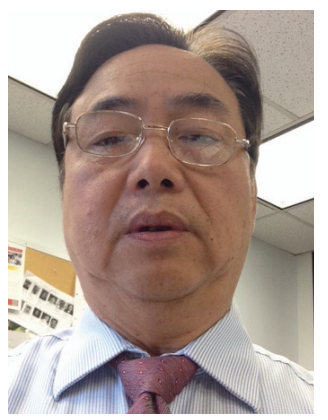

Ke-He Ruan

Author for correspondence: The Center for Experimental Therapeutics \& Pharmacoinformatics, Department of Pharmacological \& Pharmaceutical Sciences, College of Pharmacy, University of Houston, Houston, TX 77204, USA Tel.: + 17137431771 Fax: + 17137431884 khruan@uh.edu 
life span. These results have led us to hypothesize that the conflicting evidence regarding the neuronal protection induced by NSAIDs is likely a consequence of nonspecifically reduced COX-1 and -2 activities, effectively shutting down inflammatory $\mathrm{PGE}_{2}$ production, as well as that of beneficial prostanoids, such as $\mathrm{PGI}_{2}$. To help shed light on whether NSAID treatment and a resulting decrease in $\mathrm{PGI}_{2}$ could precipitate neuroinflammation, the aforementioned transgenic mice, which express increased $\mathrm{PGI}_{2}$ biosynthesis, can be used as a testing model. The neuroprotective effects of redirecting AA metabolism to $\mathrm{PGI}_{2}$ production (thus reducing inflammatory $\mathrm{PGE}_{2}$ and $\mathrm{TXA}_{2}$ production), could be assessed in vitro through the use of a co-culture protocol. Primary neuronal cell cultures from transgenic mice and microglial cells could be grown together to simulate native tissue composition. $\beta$-amyloid peptide fragment could then be used to induce neuronal cell damage and death, with MAP-2 and CD-68 serving as markers for neurons and microglia, respectively. The degree of neurodegeneration could then be determined by calculating the percentage of viable neuronal cells postexposure [20].

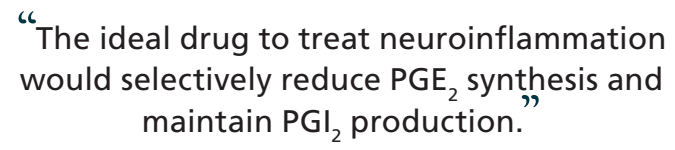

To determine whether the anti-neuroinflammatory effects seen in NSAID treatment are due to a reduction in $\mathrm{PGE}_{2}$, two methods are under testing to reconstruct the inflammatory environment in vitro. One method is to apply $\mathrm{PGE}_{2}$ directly to neuronal cells. The other method involved the use of newly created transgenic cell lines, which express the hybrid enzyme COX-2-linked to mPGES-1 hybrid enzyme. This hybrid enzyme would redirect AA metabolism to produce excess inflammatory $\mathrm{PGE}_{2}$, thus mimicking the inflammatory environment. Again, neuronal viability could be measured to compare the fluctuation in neuroinflammatory response resulting from administration of $\mathrm{PGE}_{2}$ and NSAIDs.

To address the potential benefit of $\mathrm{PGI}_{2}$ in neurodegeneration in vivo, we propose establishing a mouse

\section{References}

1 Nomura DK, Morrison BE, Blankman JL et al. Endocannabinoid hydrolysis generates brain prostaglandins that promote neuroinflammation. Science 334(6057), 809-813 (2011).

2 Rao JS, Kellom M, Kim HW, Rapoport SI, Reese EA. Neuroinflammation and synaptic loss. Neurochem. Res. 37(5), 903-910 (2012).

3 Rao JS, Rapoport SI, Kim HW. Altered neuroinflammatory, arachidonic acid cascade and synaptic markers in postmortem Alzheimer's disease brain. Transl. Psychiatry 1, e31 (2011). strain through crossbreeding hybrid enzyme expressing transgenic mice with a standard Alzheimer's mouse strain.

The ideal drug to treat neuroinflammation would selectively reduce $\mathrm{PGE}_{2}$ synthesis and maintain $\mathrm{PGI}_{2}$ production. Such a drug is not currently available. This highlights the need to search for novel lead compounds that specifically inhibit the activity of downstream mPGES-1, which is a key enzyme responsible for the synthesis of inflammatory $\mathrm{PGE}_{2}$, while retaining $\mathrm{PGI}_{2}$ synthase activity. These compounds could serve to overcome the side effects of NSAID treatment regimens, inhibiting upstream COX enzymes. Lead compounds obtained through high-throughput screening could be used as a template to design and synthesize modified compounds exhibiting therapeutic activity. Our engineered hybrid enzyme which links COX-2 to mPGES-1 mimics the coupling of inducible COX-2 and mPGES-1, could thus be used as a target in lead compound optimization [21].

In this editorial, have discussed novel approaches to elucidate the controversies surrounding the use of NSAIDs in neuroinflammation and neurodegenerative disease. We propose developing transgenic mouse and hybrid enzyme constructs as a research platform to help further our understanding of NSAIDs and their potential application in this pathology. The platform will also be valuable in optimizing novel lead compounds targeting mPGES-1, which reduces inflammation with higher specificity. With these strategies, we hope to unlock the therapeutic potential of NSAIDs in neuroinflammation and neurodegenerative disease.

\section{Financial \& competing interests disclosure}

The authors have no relevant affiliations or financial involvement with any organization or entity with a financial interest in or financial conflict with the subject matter or materials discussed in the manuscript. This includes employment, consultancies, honoraria, stock ownership or options, expert testimony, grants or patents received or pending, or royalties.

No writing assistance was utilized in the production of this manuscript.

4 Schwartz M, Shechter R. Systemic inflammatory cells fight off neurodegenerative disease. Nat. Rev. Neurol. 6(7), 405-410 (2010).

5 Hernan MA, Logroscino G, Garcia Rodriguez LA. Nonsteroidal anti-inflammatory drugs and the incidence of Parkinson disease. Neurology 66(7), 1097-1099 (2006).

6 Mcgeer PL, Mcgeer EG. NSAIDs and Alzheimer disease: epidemiological, animal model and clinical studies. Neurobiol. Aging 28(5), 639-647 (2007).

7 Schwartz M, Ziv Y. Immunity to self and self-maintenance: what can tumor immunology teach us about ALS and 
Alzheimer's disease? Trends Pharmacol. Sci. 29(6), 287-293 (2008).

8 Akitake Y, Nakatani Y, Kamei D et al. Microsomal prostaglandin E synthase-1 is induced in Alzheimer's disease and its deletion mitigates Alzheimer's disease-like pathology in a mouse model. J. Neurosci. Res. 91(7), 909-919 (2013).

9 Andreasson K. Emerging roles of PGE2 receptors in models of neurological disease. Prostaglandins Other Lipid Mediat. 91(3-4), 104-112 (2010).

Kuroki Y, Sasaki Y, Kamei D et al. Deletion of microsomal prostaglandin E synthase-1 protects neuronal cells from cytotoxic effects of beta-amyloid peptide fragment 31-35. Biochem. Biophys. Res. Commun. 424(3), 409-413 (2012).

11 Wu L, Wang Q, Liang X, Andreasson K. Divergent effects of prostaglandin receptor signaling on neuronal survival. Neurosci. Lett. 421(3), 253-258 (2007).

12 Cazevieille C, Muller A, Bonne C. Prostacyclin (PGI2) protects rat cortical neurons in culture against hypoxia/ reoxygenation and glutamate-induced injury. Neurosci. Lett. 160(1), 106-108 (1993).

13 Doi Y, Minami T, Nishizawa M, Mabuchi T, Mori H, Ito S. Central nociceptive role of prostacyclin (IP) receptor induced by peripheral inflammation. Neuroreport 13(1), 93-96 (2002).

14 Vollert C, Ohia O, Akasaka H, Berridge C, Ruan KH, Eriksen JL. Elevated prostacyclin biosynthesis in mice impacts memory and anxiety-like behavior. Behav. Brain Res. 258, 138-144 (2014).
15 Fuster V, Sweeny JM. Aspirin: a historical and contemporary therapeutic overview. Circulation 123(7), 768-78 (2011).

16 Liu Q, Xi Y, Terry T et al. Engineered endothelial progenitor cells that overexpress prostacyclin protects vascular cells. $J$. Cell. Physiol. 227(7), 2907-2916 (2012).

17 Ruan KH, So SP, Cervantes V, Wu H, Wijaya C, Jentzen RR. An active triple-catalytic hybrid enzyme engineered by linking cyclooxygenase isoform-1 to prostacyclin synthase that can constantly biosynthesize prostacyclin, the vascular protector. FEBS J. 275(23), 5820-5829 (2008).

18 Lin H, Yuan J, Ruan KH et al. COX-2-10aa-PGIS gene therapy improves erectile functions in rats after cavernous nerve injury. J. Sex. Med. 10(6), 1476-1487 (2013).

19 Ruan KH, Mohite AJ, So SP. Resistant to thrombosis, induced stroke and heart arrest by incorporation of a single gene of PGI2-synthesizing COX-1-PGIS in vivo: implication against human heart disease. Int. J. Cardiol. 168(3), 2960-2961 (2013).

20 Lehmann SM, Kruger C, Park B et al. An unconventional role for miRNA: let-7 activates Toll-like receptor 7 and causes neurodegeneration. Nat. Neurosci. 15(6), 827-835 (2012).

21 Ruan KH, Cervantes V, So SP. Engineering of a novel hybrid enzyme: an anti-inflammatory drug target with triple catalytic activities directly converting arachidonic acid into the inflammatory prostaglandin E2. Protein Eng. Des. Sel. 22(12), 733-740 (2009). 